\title{
Reliability and Performance of General Two-Dimensional Broadcast Wireless Network
}

\author{
Xiaomin $\mathrm{Ma}^{\mathrm{a}}$, Kishor S. Trivedi ${ }^{\mathrm{b}}$ \\ ${ }^{a}$ College of Science and Engineering, Oral Roberts University, Tulsa, OK 74171. E-mail: \\ xma@oru.edu \\ ${ }^{b}$ Department of Electrical and Computer Engineering, Duke University, Durham, NC \\ 27708.E-mail:kst@ee.duke.edu
}

\begin{abstract}
In this paper, an analytic model is built to study the medium access control (MAC) performance and reliability of general two-dimensional (2-D) IEEE 802.11 based wireless networks for one-hop broadcast communications. First, a semi-Markov process (SMP) model is introduced to capture the medium contention and backoff behavior and the impact of hidden terminal problem in IEEE 802.11 broadcast wireless networks in a more precise way. An $\mathrm{M} / \mathrm{G} / 1 / \mathrm{K}$ queue is used to model arrival and service of messages in an individual mobile terminal. To the contention for the shared medium, the SMP model interacts with the $\mathrm{M} / \mathrm{G} / 1 / \mathrm{K}$ queue through fixed-point iteration. Furthermore, based on the fixed-point solution, performance metrics including packet delivery probability (PDP), MAC-level and Application-level transmission delay, and MAC-level and Application-level packet reception ratio (PRR) are derived. The 2-D PDPs are derived through a coverage area computation for the impact of hidden terminal problem and concurrent transmissions to evaluate point-to-point reception probability, and then through integration of the reception probabilities over the circular intended range of the sending node. The analytical model takes into account IEEE 802.11 backoff counter process, hidden terminal problem, message inter-arrival interval, distinct transmission range, carrier sensing and interference ranges, and Nakagami fading channel with distance dependent path loss. Finally, as an example, the proposed analytic model is applied to a 2-D ad hoc network. The new model is validated through extensive simulations and new observations about the effects of network parameters on the performance and reliability are obtained.
\end{abstract}

Preprint submitted to Performance Evaluation

September 1, 2015

(C) 2015. This manuscript version is made available under the Elsevier user license http://www.elsevier.com/open-access/userlicense/1.0/ 
Keywords: Wireless networks, IEEE 802.11, General two-dimensional broadcast, hidden terminal problem, fading and path loss, medium access control

\section{Introduction}

Wireless networks such as mobile ad hoc networks (MANETs) and wireless sensor networks deployed in desert battlefields, underwater, big square fields, and big forests can be abstracted or approximated as general twodimensional (2-D) networks [1][29-30][34]. Broadcast services in such networks are provided widely in various wireless network applications such as safety applications in vehicle-to-vehicle communication [1][7], military battle field communication [3][6][16][30], and medical monitoring [2]. IEEE 802.11 based physical design and network protocols have been very commonly adopted in such wireless networks. These applications require highly reliable and real-time communications between mobile nodes under adverse environments. Modeling and analysis of IEEE 802.11 based 2-D broadcast networks plays an important role in the design and development of such networks for specific applications.

\subsection{Related Work}

There has been much research work on the performance analysis of IEEE 802.11 based wireless networks [4-5][35] and sensor networks [36]. These analyses started from Bianchi's original work in [35]. Then the work was extended to fit various different applications under different communication environments. However, most of the analytic models assumed either one-hop node spatial situations where nodes in the network can hear each other or one-dimensional Poisson node distribution. Several papers investigated the impact of the spatial distributions on the performance of networks [37-38]. However, analyses of broadcast services in the wireless networks are quite different from that in unicast systems [9]. For example, Hidden terminal area in broadcast networks is bigger than that in unicast network, and has to be evaluated in different ways [9]. Normally, the performance of broadcast networks is evaluated by simulations. In [8][11-13], analytical models are proposed to obtain PRR expressions in one-dimensional (1-D) IEEE 802.11 based broadcast ad hoc networks with hidden terminals. However, the impact of fading channel, if any, is approximated by a constant bit error rate (BER). Assuming spatially Poisson distributed nodes and saturated packet 
generation condition, $P R R$ of beacon message broadcast in vehicular ad hoc networks is investigated in [11] taking into account the impact of Rayleigh fading. Unfortunately, very few network scenarios in real applications can be abstracted as 1-D models. However, extension of 1-D network reliability analysis to general 2-D network reliability analysis is not trivial. The precise computation of expected potential hidden terminal area given a random distribution of nodes in 2-D area is still claimed to be an open problem although some approximations have been made to approach the evaluation [9][10]. Recently, we conducted $P R R$ analysis in a special type of 2-D ad hoc networks (two parallel lines approximate two opposite roads on highway) [14][15] and a general 2-D network with ideal communication environment [27]. An Markov chain model was proposed for the analysis of multi-hop unicast networks with Rayleigh fading channel in [23]. As of now, there is no work on the performance evaluation of general 2-D IEEE 802.11 broadcast networks taking real factors and adverse communication environments into account. Furthermore, MAC level or network level performance focusing on success of packet by packet transmissions, sometimes, is not sufficient to characterize the networks from application perspective [39] [40]: the network 's ability for all intended mobile nodes to receive the broadcast messages within specified operation duration is more concerned about.

\subsection{Our Contributions}

In this paper, we propose a new analytic model for the analysis of general 2-D and IEEE 802.11 based broadcast networks including all the mentioned features. Compared with the existing models for performance analysis of broadcast in the wireless networks, the main contributions of the proposed analytic model in this paper are: 1) Instead of calculating broadcast hidden terminal area directly, a new approach through integration of point-to-point packet delivery probability over intended 2-D area is proposed to derive the one-hop broadcast reliability and transmission delay accounting for IEEE 802.11 MAC, non-saturated packet generation, hidden terminal problem, and fading channel with path loss. 2) The impact analysis of distance related Nakagami fading on the performance is conducted; 3) Introducing semi-Markov process (SMP) model [20] interacting with $\mathrm{M} / \mathrm{G} / 1 / \mathrm{K}$ queue model facilitates the accurate evaluation of IEEE 802.11 broadcast and hidden terminal impact that is one of the major factors leading to the degradation of the reliability; 4) Application-level metrics are derived from the MAC-level performance and reliability; 5) Different from the existing PRR expressions, 
which are average metrics among all receivers within sender's transmission ranges, the new $P R R$ in this paper are functions of the receivers' distances to the broadcast sender, which provides a deeper insight into the performance as the distances vary.

This paper is organized as follows. Section 2 presents a brief description of IEEE 802.11 broadcast MAC, broadcast hidden terminal problem, channel fading with path loss in wireless network environment, and assumptions under which the analytic model is built. Section 3 presents SMP analytic models and the fixed-point iteration algorithm. Consequently, performance metrics such as mean transmission delay, packet delivery probability, and packet reception ratio in both MAC-level and application-level are derived in the 2-D IEEE 802.11 broadcast wireless networks. Section 4 demonstrates and discusses the numerical results from the analytic model and the simulation. The paper is concluded in Section 5.

\section{Description of Wireless Network Broadcast and Environment and Assumptions}

\subsection{Distributed Coordination Function for IEEE 802.11 Broadcast Service}

MAC layer of IEEE 802.11 [1] deploys a random access scheme for all associated devices in a cluster based on carrier sense multiple access with collision avoidance (CSMA/CA). In the 802.11 MAC protocols, the fundamental mechanism for medium access is the distributed coordination function (DCF). DCF is meant to support an ad hoc network without the need for any infrastructure elements such as an access point. Broadcast procedure of 802.11 MAC follows the basic medium access protocol of DCF. Broadcast of wireless network MAC occurs when a broadcast packet arrives at wireless network MAC layer from the upper layer and the MAC senses the channel status first and stores the status. Next, once an idle period is equal to DCF inter-frame space (DIFS) or extended inter-frame space (EIFS) (If previously received frame or packet contains error, before transmitting a frame, it has to defer EIFS duration. Otherwise, DIFS delay is deferred) is observed, MAC takes the next operation according to the stored channel status and the current value of its backoff time. If the current value of the backoff counter is not zero, MAC begins the backoff countdown process. If the current value of backoff counter is zero, MAC generates a random backoff time and begins the backoff countdown process. If the backoff counter counts down to 
zero, MAC begins the packet transmission immediately. During the backoff countdown process, carrier-sense persists. If the medium becomes busy again, MAC goes back to the DIFS/EIFS observation process. During or after a broadcast transmission, MAC does not monitor the success or failure of the transmission. Once transmission completes, MAC simply releases the medium and competes for it when a new packet is ready to be sent.

\subsection{Radio Channel Fading and Path Loss in Wireless Networks}

Wireless networks present scenarios with unfavorable characteristics to develop wireless communications with multiple reflecting objects able to degrade the strength and quality of the received signal. Additionally, fading effects resulting from the mobility of the surrounding objects and/or the sender and receivers themselves have to be taken into account. Wireless channel modeling involves two important aspects [16]: large scale path loss and small scale fading. The former is used to determine the average received signal strength at a particular distance from the transmitter, whereas small scale fading generally involves the detailed modeling of multi-path fading statistics, power delay profile, and Doppler spectrum. Several studies [6] have demonstrated that the probability density function (pdf) of signal amplitude $X_{a}$ at a given distance in wireless channels is well described by the two-parameter Nakagami density:

$$
f\left(x_{a}, \omega, m\right)=\frac{2 m^{m} x_{a}^{2 m-1}}{\Gamma(m) \omega^{m}} \exp \left(\frac{m x_{a}{ }^{2}}{\omega}\right), x_{a} \geq 0, \omega>0, m \geq 1 / 2
$$

where $\Gamma()$ is the standard Gamma function, $m$ is the fading parameter, and $\omega$ is the average received power. The values of the two parameters are functions of distance. According to above density function, the probability density function (pdf) of power $X=X_{a}{ }^{2}$ (square of the signal amplitude) at the given distance is derived by a change of variables and is given by a gamma distribution of the following form:

$$
f_{d}(x, \omega, m)=\frac{m^{m}}{\Gamma(m) \omega^{m}} x^{m-1} e^{-\left(\frac{m x}{\omega}\right)}
$$

Since the received power is clearly a function of distance from the sender, the path loss model is represented by the following equation [21]:

$$
\frac{\omega\left(r_{0}\right)}{\omega(r)}=\left(\frac{r}{r_{0}}\right)^{\gamma}
$$


where $\omega\left(r_{0}\right)$ and $\omega(r)$ are the mean received power with distance to the sender $r_{0}$ and $r$, respectively, and $\gamma$ is the path loss exponent, which is usually empirically determined by field measurements. Normally, $\gamma$ can be 2 for free space environment, 1.6 1.8 for indoor line of sight, and can be $2.7 \sim 5$ for obstructed area or shadowed urban area [21].

\subsection{Hidden Terminal Problem in Broadcast}

A multi-hop ad hoc (or sensor) network has the advantage that multiple concurrent transmissions can take place simultaneously at geographically separated locations. However, such a capacity gain may be offset by the hidden terminal problem. Hidden terminals are two terminals, though outside the interference range of one another, share a set of terminals that are within the transmission ranges of both. The problem of hidden terminals is a critical factor in the performance of ad hoc (or sensor) networks. Although the support for reliable unicast using RTS/CTS has existed traditionally in IEEE 802.11 [1], there has not been any MAC-level recovery or retransmission of frames for reliable broadcasting. Since broadcast in IEEE 802.11 does not use virtual carrier sensing and thus only relies on physical carrier sensing to reduce collisions, the potential hidden terminal area of a sending node includes the receiving range of all the nodes within the transmission range of the sender. Thus, the potential hidden terminal area in the broadcast can be considerably larger than that in unicast [8].

\subsection{Assumptions for IEEE 802.11 2-D Broadcast Networks}

a) We consider a wireless broadcast network with nodes distributed randomly according to the two-dimensional Poisson distribution. Let the average number of nodes per square unit (density) in the network be $\beta$. The probability $P(i, s)$ of finding $i$ nodes in an area of $s$ units is given by

$$
P(i, s)=\frac{(\beta s)^{i} e^{-\beta s}}{i !}
$$

For networks where transmitters and/or receivers are located or move around randomly over a large area, the Poisson point process is a good approximation [17]. It corresponds to a uniform distribution for infinite networks.

b) All nodes have the same deterministic transmission range $R$, same carrier sensing range $L_{c s}, R \leq L_{c s} \leq 2 R$, and same interference range $L_{\text {int }}$, $R \leq L_{\text {int }} \leq L_{c s}$. Deterministic communication ranges approximate the 
average of random communication ranges, which is validated by extensive simulations using transmission powers and receiving thresholds

c) At each mobile node, packet arrivals follow a Poisson process with rate $\lambda$ (in packets per second).

d) Each node has a finite queue with queue length $K$ (including the one in service) to store the packets at the MAC layer.

e) Impacts of node mobility on the performance are not considered in the paper. It has been proved in [28] that high node mobility has minor impact on the performance of the one-hop direct message broadcast network with high data transmission rates

f) Nakagami fading channel with path loss is assumed for studying the impact of imperfect channel on the performance [6].

g) The temporal interaction between nodes is not captured in the theoretical model, which is reduced to a busy probability.

\section{System Model and Performance Analysis}

\subsection{SMP Model for IEEE 802.11 Broadcast}

Next, we try to develop a model to characterize and evaluate the communication system for channel access and message broadcast. Due to complexity of the contention medium and channel access service, the service process cannot be assumed to be Poisson. So, based on above understanding of the system and the assumptions, the overall problem can be seen as a set of interacting $\mathrm{M} / \mathrm{G} / 1 / \mathrm{K}$ queues, one queue for each node. We simplify the problem by developing an semi-Markov process (SMP) model for the tagged node that does not directly keep track of the queued requests but captures the channel contention and IEEE 802.11 backoff behavior. Compared with the discrete time Markov chain (DTMC) model adopted for the system in the previous research [8] [12], the SMP model can accurately capture the continuous time system behavior beyond the per-slot statistics. To facilitate the tractability of the model, the behavior of a tagged node for channel access and packet transmission is approximated by the SMP model interacting with a set of $\mathrm{M} / \mathrm{G} / 1 / \mathrm{K}$ queues. This SMP model interacts with the $\mathrm{M} / \mathrm{G} / 1 / \mathrm{K}$ queues of the other nodes through fixed-point iteration.

As shown in Fig. 1. The tagged node is in idle state if there is no packet in its queue. After a packet is generated, the node senses channel activity for DIFS time period. Next, the node will randomly choose a backoff counter 


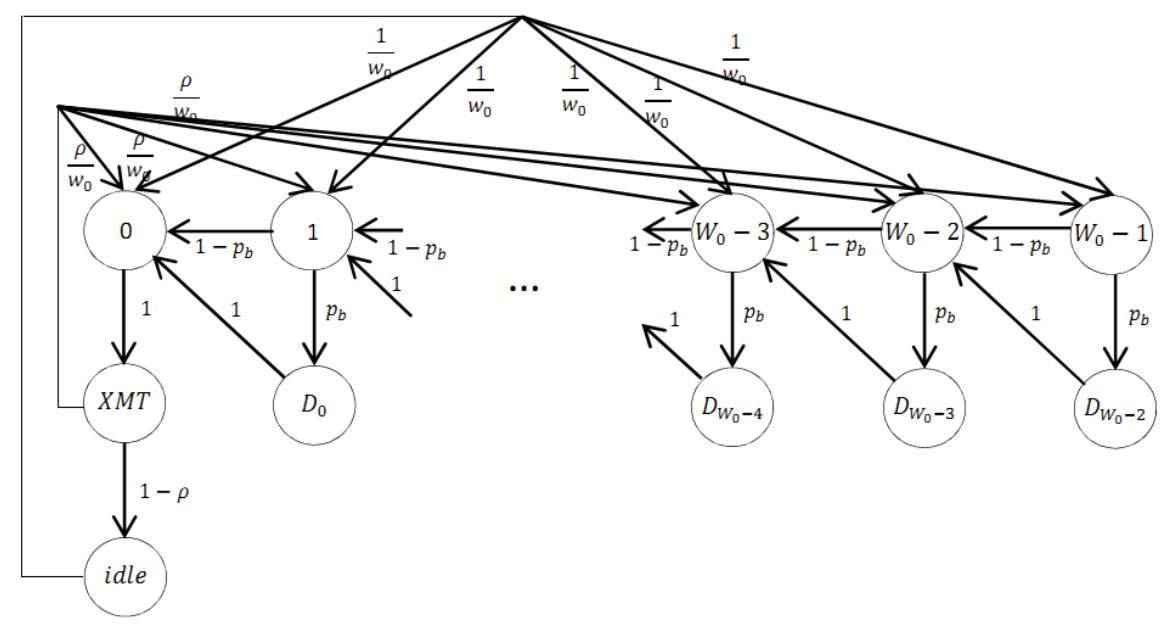

Figure 1: SMP model for IEEE 802.11 broadcast

in the range $\left[0, W_{0}-1\right]$. The backoff counter will be decreased by one if the channel is detected to be idle for a time slot $\sigma$ (with probability $1-p_{b}, p_{b}$ is defined as the probability that the channel is detected busy in one time slot by the tagged node), which is captured by the transition from $W_{0}-i$ to $W_{0}-i-1$ state. If the channel is busy during a backoff time slot $\sigma$, the backoff counter of the tagged node will be suspended and deferred for the duration $T$ that includes a packet transmission time and an idle DIFS, which presents the transition from state $W_{0}-i$ to state $D_{W_{0}-i-1}$ with probability $p_{b}$. When the backoff counter reaches zero, the packet will directly be transmitted (an SMP transition occurs from 0 state to state $X M T$ with probability one). In $X M T$ state, a packet is transmitting. After the packet transmission, if there is no packet left in the queue of the tagged node (with probability 1- $\rho$, where $\rho$ is defined as the probability that there are packets in the queue of the tagged node), the node will go from XMT to idle state and wait for a new incoming packet. If there are packets left in the queue after a packet transmission (with probability $\rho$ ), the node will sense the channel again for DIFS time and then randomly choose a backoff counter before transmitting the next packet. The fact that we simply use server utilization as a surrogate for the queue of waiting messages is a key approximation. Notice that the SMP model in Fig. 1 is a simplified version of the SMP model in [20] ( $q_{b}$ is equal to 1 in this paper) considering that the possibility of a packet transmission without going through backoff process is very small and negligible. 
Define the sojourn time in state $j$ as $T_{j}$. The mean and variance of $T_{j}$ in the SMP model are:

$$
E\left[T_{j}\right]=\tau_{j}= \begin{cases}\sigma & j=0,1,2, \ldots, W_{0}-1 \\ T & j=D_{0}, D_{1}, \ldots, D_{W_{0}-2} \\ T & j=X M T \\ \frac{1}{\lambda}+\text { DIFS } & j=i d l e\end{cases}
$$

where $T=\frac{E[P A]}{\left(R_{d} \times 10^{6}\right)}+T_{H}+D I F S+\delta$ The mean of the packet transmission packet size $P A$ is $E[P A]$. $R_{d}$ presents the data rate in Mbps. Hence, $\frac{E[P A]}{\left(R_{d} \times 10^{6}\right)}$ is the time to transmit the packet. $T_{H}$ presents the packet header time including physical layer header and MAC layer header. $\delta$ is the propagation delay. EIFS = Transmission time of Ack frame at lowest basic rate $+S I F S+D I F S$, where SIFS is time duration for Short Interframe Space. Although EIFS is much longer than DIFS, EIFS waiting duration only occurs occasionally when low transmission error rate is assumed in the applications that require reliable delivery of messages. On the other hand, there is no Ack frame in IEEE 802.11 broadcast. So, we assume in our model that the impact of EIFS can be neglected.

For the SMP model in Fig. 1, the embedded DTMC is first solved for its steady-state probabilities:

$$
b_{i}= \begin{cases}\left(W_{0}-j\right) b_{W_{0}-1}, & j=0,1,2, \ldots, W_{0}-1 \\ \left(W_{0}-j-1\right) p_{b} b_{W_{0}-1} & j=D_{0}, D_{1}, \ldots, D_{W_{0}-2} \\ W_{0} b_{W_{0}-1} & j=X M T \\ W_{0}(1-\rho) b_{W_{0}-1} & j=i d l e\end{cases}
$$

In the above equations

$$
b_{W_{0}-1}=\frac{2}{\left(1+p_{b}\right) W_{0}^{2}+\left(5-p_{b}-2 \rho\right) W_{0}}
$$

Taking into account the mean sojourn time in each state, the steady-state probabilities of the semi-Markov process are given by:

$$
\pi_{i}=\frac{b_{i} \tau_{i}}{\sum_{j} b_{j} \tau_{j}}
$$

Therefore, the steady-state probability that a node is in the $X M T$ state is given

$$
\pi_{X M T}=\frac{2 T}{\left(\sigma+p_{b} T\right) W_{0}+\sigma-p_{b} T+2 T+2(1-\rho)\left(\frac{1}{\lambda}+D I F S\right)}
$$


Although the sojourn time in $X M T$ state is $T$, the real packet transmission only occupies a portion of this sojourn time, which is $\frac{E[P A]}{\left(R_{d} \times 10^{6}\right)}+T_{H}+\delta=$ $T-D I F S$. Hence, the probability that a node is transmitting in steady state is $\pi_{X M T} \frac{(T-D I F S)}{T}$.

In Equation (8), two unknown parameters are: $\rho$ and $p_{b}$. It is easy to see that $p_{b}$ of the tagged node depends on the transmitting state of other nodes within the tagged node's receiving range, and the transmitting probability of a node can be described by Equation (8) (as a function of $p_{b}$ and $\rho$ ). Hence, $p_{b}$ of the tagged node can be expressed as a function of $p_{b}$ and $\rho$ of other nodes. Clearly, we have made the homogenity assumption for all the nodes. In addition, we know that $\rho$ depends on the mean service time to transmit a packet. Therefore, the service time is derived first in the next subsection.

\subsection{Service Time Computation}

As mentioned above, each node in the network is modeled as an $\mathrm{M} / \mathrm{G} / 1 / \mathrm{K}$ queue. The MAC layer service time is defined as the time interval from the time instant when a packet becomes the head of the queue and starts to contend for transmission, to the time instant when the packet is received.

The SMP model in Section 3.1 describes the behavior of a tagged node transmitting packets in its queue. This SMP model takes the state of its own queue into account by means of a surrogate $\rho$. Hence, at the end of one packet transmission, it checks whether there are packets left in its queue or not to transmit the next packet. In this section, the service time for any one packet in the queue is derived. Therefore, the SMP model in Section 3.1 is modified to contain an absorbing state END as shown in Fig. 2. Thus, at the end of one packet transmission, the SMP model reaches an absorbing state instead of checking the status of the queue to transmit the next packet. By proper choice of the initial probability vector, the time to reach the absorbing state will be the service time for a packet transmission.

For a newly generated packet in the tagged node, the node will randomly choose a backoff counter before the packet transmission. Therefore, the initial probability that a packet starts its service from state $i\left(i=0,1, \ldots, W_{0}-1\right)$ is $\frac{1}{W_{0}}$. Hence, the initial probability vector is:

$$
q_{i}= \begin{cases}\frac{1}{W_{0}} & i=0,1,2, \ldots, W_{0}-1 \\ 0, & i=D_{0}, D_{1}, D_{2}, \ldots, D_{W_{0}-2}, X M T\end{cases}
$$

Since the embedded DTMC contains an absorbing state (state END in Fig. 2), taking advantage of the acyclic nature of the DTMC model in Fig. 

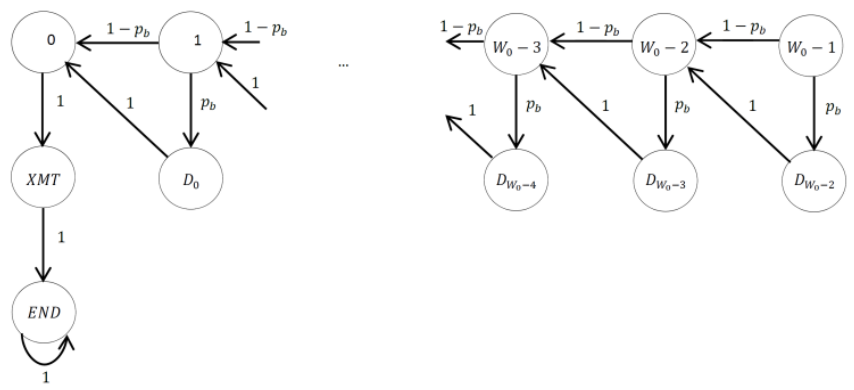

Figure 2: Embedded DTMC of the SMP model for the service time

2, the mean service time for a packet transmission conditioned on starting from state $i$ is derived from:

$$
E\left[S_{i}\right]=i \sigma+i p_{b} T+T \quad \text { for } \quad i=0,1, \ldots, W_{0}-1
$$

Therefore, the mean of the service time is given by

$$
E[S]=\sum_{i} E\left[S_{i}\right] q_{i}=\frac{\left(\sigma+p_{b} T\right)\left(W_{0}-1\right)}{2}+T
$$

\subsection{Fixed-point Iteration}

From the tagged node's point of view, $p_{b}$ is the probability that it senses channel busy during one time slot in the backoff process. Since channel is detected busy if there is at least one neighbor (i.e., a node in the transmission range of the tagged node) transmitting in a backoff time slot of the tagged node, we have

$$
p_{b}=1-\sum_{i=0}^{\infty}\left(1-P_{X M T}\right)^{i} \frac{\left(\beta \pi L_{c s}^{2}\right)^{i}}{i !} e^{-\beta \pi L_{c s}^{2}}=1-e^{-\beta \pi L_{c s}^{2} P_{X M T}}
$$

where $P_{X M T}$ is the probability that a neighbor is transmitting in a backoff time slot of the tagged node, to be derived later in Eq.(13).

For the first backoff time slot of the tagged node, the time duration that can capture the transmission of the neighbor is $T-D I F S+2 \sigma$. One extra time slot $\sigma$ is the one just before transmission and another is the one just after transmission, which can capture the starting time instant and ending time instant of the packet transmission. Therefore, the probability that a 
neighbors' transmission is detected in the first backoff time slot of the tagged node is $\pi_{X M T} \frac{(T-D I F S+2 \sigma)}{T}$.

For a backoff time slot that is not the first backoff time slot of the tagged node, the time duration that captures the transmission of the neighbor is $2 \sigma$ [20]. Therefore, the probability that a neighbors' transmission is detected in non-first backoff time slot of the tagged node is $\pi_{X M T} \frac{2 \sigma}{T}$.

Since the probability that a backoff time slot is the first backoff time slot is $\frac{1}{W_{0}}$ and non-first backoff time slot is $\left(1-\frac{1}{W_{0}}\right)$, the probability that a neighbor 's transmission is detected by a backoff time slot of the tagged node is given by

$$
P_{X M T}=\frac{1}{W_{0}} \frac{T-D I F S+2 \sigma}{T} \pi_{X M T}+\left(1-\frac{1}{W_{0}}\right) \frac{2 \sigma}{T} \pi_{X M T}
$$

In the previous section, the mean service time $E[S]$ is shown to depend on $p_{b}$. And $\rho$ in turn depends on the mean service time as per the $M / G / 1 / K$ queue equation $\rho=\lambda E[S]$. Also,from the analysis of the relationship between two parameters $\rho$ and $p_{b}$ in (12) and (13), we notice that $p_{b}$ depends on $\rho$ and $p_{b}$ itself. Because of this cyclic dependence among the parameters, fixedpoint iteration algorithm is utilized to obtain the final converged solutions. Hence, we denote $p_{b}=g\left(\rho, p_{b}\right)$ and the reciprocal of mean service time for $\mathrm{M} / \mathrm{G} / 1 / \mathrm{K}$ queue to be $\mu=h\left(\rho, p_{b}\right)$. The fixed-point iteration algorithm is outlined below.

Fixed-point iteration algorithm:

Step 1: Initialize $\rho=1$, which is the saturation condition;

Step 2: With $\rho$, solve $p_{b}$ using Equations. (8)(12)(13);

Step 3: With $\rho$ and $p_{b}$, calculate service rate $\mu=1 / E[S]$ using Equation (11);

Step 4: If $\lambda<\mu, \rho=\frac{\lambda}{\mu}$; otherwise, $\rho=1$;

Step 5: If $\rho$ converges, then stop the iteration. Otherwise, go to step 2 with the updated $\rho$.

By utilizing the fixed-point iteration, the parameters $\rho, p_{b}, \pi_{X M T}$ as well as the mean of the service time are determined, which are subsequently used for the performance indices computation in the next section. The convergence and uniqueness of the fixed-point iteration are theoretically guaranteed although their proofs cannot be demonstrated here due to space limitation. 


\subsection{MAC-level Performance Indices}

\subsubsection{Packet Transmission Delay}

The packet transmission delay is defined as the average delay a packet experiences from the time at which the packet is generated, and the time at which the packet is successfully received by all neighbors of the node that generates the packet. The transmission delay $E[D]$ includes the queuing delay and medium service time (due to backoff, packet transmission, and propagation delay, etc.).

Based on the assumptions in Section 2.4, the queuing system can be modeled as M/G/1/K. Following [26], we define $\zeta(t)(t \geq 0)$ be the state of the queuing system at time $t$. The state space of $\zeta(t)$ can then be expressed as

$$
S_{\zeta}=\left\{I, A_{0} \cdot A_{1}, \ldots, A_{K}\right\}
$$

where $A_{k}$ means that the channel server is busy and there are $k$ packets waiting in the queue; $I$ means the channel server is idle, or in other words, the queue and the transmission buffer are empty. We now consider an embedded Markov process that has state space $S^{\prime}=S-I=\left\{A_{0}, A_{1}, A_{2}, \ldots, A_{K}\right\}$. The Markov chain is embedded to departure instants. Let $p_{i j}$ be the transition probability from state $A_{i}$ to $A_{j}(0 \leq i, j \leq K)$

$$
p_{i j}=\lim _{n \rightarrow \infty} \operatorname{Pr}\left\{\zeta_{n+1}=A \mid \zeta_{n}=A_{i}\right\}
$$

To obtain $p_{i j}$, we define $\alpha(k)$ as the probability that $k$ packets have arrived during one packet service time $E[S]$. From Fig.2 in the paper, we have probability mass function (pmf) of $S_{i}$

$$
\begin{aligned}
& S_{i}=i \sigma+m T+T \text {, with probability } \\
& \qquad P_{s}(m)=C_{i}{ }^{m} p_{b}{ }^{m}\left(1-p_{b}\right)^{i-m}, i \geq m \geq 0
\end{aligned}
$$

where $m$ is the number of times that the channel is busy throughout the slot backoff, $C_{i}{ }^{m}$ is the binomial coefficient indexed by $m$ and $i$. Since the packet arrival process is Poisson with rate $\lambda$, we have

$$
\alpha(k)=\sum_{i=0}^{W_{0}-1} q_{i} \sum_{m=0}^{i} \frac{e^{-\lambda(i \sigma+m T+T)}[\lambda(i \sigma+m T+T)]^{k}}{k !} \times \frac{}{C_{i}{ }^{m} p_{b}{ }^{m}\left(1-p_{b}\right)^{i-m}}
$$


where $q_{i}$ is the probability that one packet service time is $S_{i}$, which can be derived from Eq. (9). Consequently, $p_{i j}$ can be obtained as

$$
P=\left\{p_{i j}\right\}=\left[\begin{array}{cccccc}
\alpha(0) & \alpha(1) & \ldots & \alpha(K-2) & 1-\sum_{k=0}^{K-2} \alpha(k) \\
\alpha(0) & \alpha(1) & \ldots & \alpha(K-2) & 1-\sum_{k=0}^{K-2} \alpha(k) \\
0 & \alpha(0) & \ldots & \alpha(K-3) & 1-\sum_{k=0}^{K-3} \alpha(k) \\
\vdots & \vdots & \ldots & \vdots & \vdots \\
0 & 0 & \ldots & \alpha(0) & 1-\alpha(0)
\end{array}\right]
$$

Since $\nu_{k}(0 \leq k \leq K)$ is the steady-state probability that $\zeta_{n}=A_{k}$, we can calculate all $\nu_{k}{ }^{s}$ by solving the embedded Markov chain with all $p_{i j}$ according to the following balance equations:

$$
\left\{\begin{array}{c}
\nu P=\nu \\
\sum_{k=0}^{K-1} \nu_{k}=1
\end{array}\right.
$$

Having solved the embedded Markov chain (see Appendix C), we obtain $\nu_{k}(0 \leq k \leq K)$. Let $P_{k}$ be the probability that there are $k$ packets in the system at an arbitrary time, which can be evaluated in terms of the steadystate probabilities [33]

$$
P_{k}=\frac{\nu_{k}}{\nu_{0}+\rho} \quad 0 \leq k \leq K-1
$$

The probability that the queue is full or blocked can be calculated as [33]

$$
P_{K}=P_{B}=1-\frac{1}{\nu_{0}+\rho}
$$

and

$$
P_{I}=P_{0}=\frac{\nu_{0}}{\nu_{0}+\rho}
$$

Where $\rho=\lambda E[S]$ is the traffic intensity. So, considering that queue delay is zero when the channel server is idle, the expected queuing delay is obtained as

$$
E\left[D_{q}\right]=\frac{\left(1-P_{I}\right)}{\lambda}\left[\sum_{k=1}^{K-1} k P_{k}+K\left(P_{0}+\rho-1\right)\right]
$$


The average packet transmission delay is then calculated as

$$
E[D]=E\left[D_{q}\right]+E[S]
$$

\subsubsection{Packet Delivery Probability}

Packet delivery probability (PDP) is defined as the probability that a node within the transmission range of the sender successfully receives a packet from the tagged node (i.e., sender).

There are three factors affecting the performance of packet reception: hidden terminal problems, collisions due to concurrent packet transmissions, and interference as a result of fading channel.

The transmission scenario is shown pictorially in Fig. 3. Given a transmitting node $O$ placed at the origin, $Q$ is one of the receivers within the transmission range (i.e., a circular coverage area having radius $R$ ) of node $O$. $Q$ is placed on the $x$ axis at a distance $r_{0}$ from $O$.

Let $V:\left(r_{1} \cos \phi, r_{1} \sin \phi\right)$ be another node in the transmission range of $O$ and $Q$. In order to evaluate the impact of hidden terminals and concurrent transmissions on $P D P$, two areas need to be considered. $S_{1}\left(r_{0}\right)=$ $D\left(Q, L_{i n t}\right)-D\left(O, L_{c s}\right)$ denotes the hidden terminal area of node $O$, where $D(s, l)$ represents the disk of radius $l$ centered at $s$. Denote $S_{2}\left(\phi, r_{1}, r_{0}\right)=$ $D\left(V, L_{c s}\right)-D\left(O, L_{c s}\right) \cup D\left(Q, L_{i n t}\right)$ as the area within the carrier sensing range of $V$ that is not in the carrier sensing range of $O$ or interference range of $Q$.

Impact of Hidden Terminals We observe that since the transmission time for a packet is $T-D I F S=\frac{E[P A]}{\left(R_{d} \times 10^{6}\right)+T_{H}+\delta}$, the transmissions from hidden terminals collide with the tagged node's transmission only happens when hidden terminals start to transmit during the vulnerability period $2(T-$ $D I F S)=2\left(\frac{E[P A]}{\left(R_{d} \times 10^{6}\right)+T_{H}+\delta}\right)$. Given the probability that a node starts to transmit during the vulnerable period is $\pi_{X M T} 2 \frac{(T-D I F S)}{T}[20]$, we have

$$
\begin{aligned}
P_{H}\left(r_{0}\right) & =\sum_{i=0}^{\infty}\left(1-\pi_{X M T} \frac{2(T-D I F S)}{T}\right)^{i} \times \frac{\beta\left|S_{1}\left(r_{0}\right)\right|^{i}}{i !} e^{-\beta\left|S_{1}\left(r_{0}\right)\right|} \\
& =\exp \left(-\frac{2(T-D I F S) \beta\left|S_{1}\left(r_{0}\right)\right| \pi_{X M T}}{T}\right) .
\end{aligned}
$$

where $\left|S_{1}\left(r_{0}\right)\right|$ is the area of $S_{1}\left(r_{0}\right)$. Derivation of $\left|S_{1}\left(r_{0}\right)\right|$ can be seen in Appendix A. 


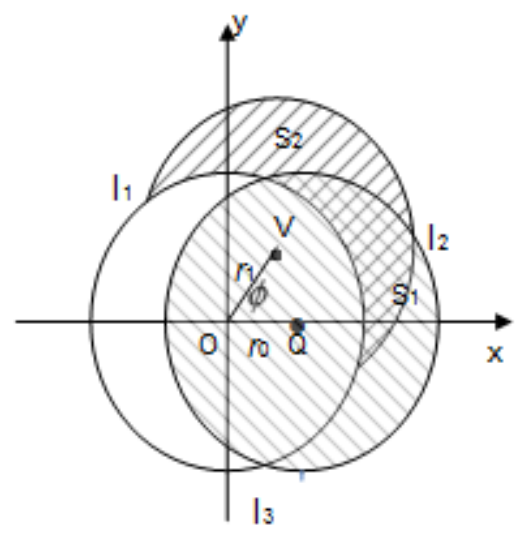

Figure 3: 2-D model for performance analysis

Impact of Concurrent Transmissions In addition to collisions caused by the hidden terminals, transmissions from nodes within the interference range of the tagged node in the meantime at which the tagged node transmits may also cause collisions. When the tagged node transmits in a slot time, concurrent collisions will take place if any node in the interference range of the tagged node transmits in the slot.

Given that both $O$ and $Q$ sense the channel idle, $V$ will transmit within the duration of a slot with probability $\tau=\pi_{0}$. It should be noted that the slots of $O$ and $V$ are not necessarily synchronized. Now, given that all nodes inside the union of two circles center $O, Q$, radius $R$ and $L_{\text {int }}$, respectively, are idle at the beginning of the slot, the probability that a node $V$ starts transmitting during the slot is the probability that node $V$ intends to transmit and all nodes in $S_{2}$ are not in the transmitting state, which is expressed as

$$
\begin{aligned}
P_{2}^{\prime}\left(\phi, r_{1}, r_{0}\right) & =\pi_{0} \sum_{i=1}^{\infty}\left(1-P_{X M T}\right)^{i} \frac{\beta\left|S_{2}\left(\phi, r_{1}, r_{0}\right)\right|^{i}}{i !} \times e^{-\beta\left|S_{2}\left(\phi, r_{1}, r_{0}\right)\right|} \\
& =\pi_{0} \exp \left(-\beta P_{X M T}\left|S_{2}\left(\phi, r_{1}, r_{0}\right)\right|\right)
\end{aligned}
$$

where $\left|S_{2}\left(\phi, r_{1}, r_{0}\right)\right|$ is the area of $S_{2}\left(\phi, r_{1}, r_{0}\right)$. Derivation of $\left|S_{2}\left(\phi, r_{1}, r_{0}\right)\right|$ can be seen in Appendix B. Then, the average number of nodes that start transmission during the slot that collides with the transmission from $O$ within 
the circle with center $Q$ and radius $L_{i n t}$ is

$$
\bar{n}_{T}=\beta \int_{0}^{2 \pi} \int_{0}^{L_{i n t}} r P_{2}^{\prime}\left(\phi, r, r_{0}\right) d r d \phi
$$

Therefore, given Poisson node distribution, the probability that no node within the circle center $Q$ radius $L_{\text {int }}$ start transmission during the slot that collides with the transmission from $O$ is

$$
P_{c o n}\left(r_{0}\right)=\frac{\left(\bar{n}_{T}\right)^{0}}{0 !} \exp \left(-\bar{n}_{T}\right)=\exp \left(-\bar{n}_{T}\right)
$$

Impact of Fading and Path Loss For the Nakagami distribution with a positive integer value for the fading parameter $m$, from (2) in Section 2.2, we obtain the corresponding cumulative distribution function (cdf) for a signal to be received with power $x$ for a given average power strength $\omega$,

$$
F_{d}(x, \omega, m)=\frac{m^{m}}{\Gamma(m) \omega^{m}} \int_{0}^{x} z^{m-1} e^{-\left(\frac{m}{\omega}\right) z} d z
$$

Then, the probability that a message is successfully received in the absence of interference deduces from the probability that the message 's signal is stronger than the reception threshold $T R_{x}$, that is,

$$
\begin{aligned}
P\left(X>T R_{x}\right) & =1-F_{d}\left(T R_{x}, \omega, m\right) \\
& =1-\frac{m^{m}}{\Gamma(m) \omega^{m}} \int_{0}^{T R_{x}} z^{m-1} e^{-\left(\frac{m}{\omega}\right) z} d z
\end{aligned}
$$

Let $z^{\prime}=\frac{z}{\omega}$, with a certain mathematical manipulation, we have

$$
P\left(X>T R_{x}\right)=1-\frac{m^{m}}{\Gamma(m)} \int_{0}^{\frac{T R_{x}}{\omega}} z^{m-1} e^{-m z} d z
$$

$T R_{x}$ should, on the average, be detected in a distance equal to the "intended" communication range $R$ from the transmitter. $\omega\left(r_{0}\right)$ should be detected as the average reception power at the distance $r_{0}$. From (3), we have:

$$
\frac{T R_{x}}{\omega\left(r_{0}\right)}=\left(\frac{r_{0}}{R}\right)^{\gamma}
$$


By applying $T R_{x}$ and $\omega\left(r_{0}\right)$ to $(26)$ we obtain the expected probability of successfully receiving a message at distance $r_{0}$ :

$$
P_{F}\left(r_{0}\right)=1-\frac{m^{m}}{\Gamma(m)} \int_{0}^{\left(\frac{r_{0}}{R}\right)^{\gamma}} z^{m-1} e^{-m z} d z
$$

Taking hidden terminal, possible packet collisions, fading with path loss, and queue blocking into account, and assuming these three events and nodes in the network are independent, the probability that the node $Q$ receives the broadcast message from the tagged node $O$ is approximated as

$$
P_{s}\left(r_{0}\right)=P_{H}\left(r_{0}\right) P_{F}\left(r_{0}\right) P_{c o n}\left(r_{0}\right) P_{B}
$$

\subsubsection{Packet Reception Ratio(PRR)}

$P R R$ is defined as the percentage of nodes that successfully receive a packet from the tagged node given that all receivers are within the transmission range of the sender at the moment that the packet is sent out [6]. From the above definition, $P R R$ can be interpreted as the percentage of the mobile nodes in the tagged node 's transmission range that receive the broadcast message successfully. We approach $P R R$ evaluation in two steps. First, compute the probability that the individual node $Q$ successfully receives the broadcast packet from the sender $O$. Then, $P R R$ is derived through integration of the probabilities over the transmission range of $O$. Assuming the distribution of nodes along a 2-D area follows Poisson process, the average number of nodes within an incremental area $d A$ should be $\beta d A$ [15]. Given the reception probability of each node in (29), the average number of nodes within $d A$ that successfully receive the broadcast message from the tagged node is $P_{s}(r) \beta d A$. For a circular coverage area having radius $R$ from node $O, P R R$ over a coverage area with radius $d(d \leq R)$ found by integrating the probability that nodes with distance $r$ to the source node $O$ within an incremental area $d A$ successfully receive the broadcast message from $O$. Hence,

$$
\operatorname{PRR}(d)=\frac{1}{\pi d^{2}} \int_{0}^{2 \pi} \int_{0}^{d} P_{s}(r) r d r d \phi=\frac{2}{d^{2}} \int_{0}^{d} P_{s}(r) r d r
$$

\subsection{Application-level Performance Indices}

\subsubsection{T-window PRR}

Application-level T-window probability is defined as the probability of successfully receiving at least one packet out of multiple packets from a broadcast node at distance $r$, within a given time $T_{w}$ (referred to as application 
tolerance window)

$$
P_{A p p}\left(r, T_{w}\right)=1-\left(1-P_{s}(r)\right)^{\frac{T_{w}}{t}}
$$

where $t=1 / \lambda$ is the packet generation interval and $P_{s}(r)$ is the node reception probability given in Eq. (29). T-window $P R R_{T}$ is defined as $P R R$ in application level. Given Application-level probability $P_{A p p}\left(r, T_{w}\right)$, we have

$$
\operatorname{PRR}_{T}(r)=\frac{2}{r^{2}} \int_{0}^{r} P_{A P P}\left(r_{0}, T_{w}\right) r_{0} d r_{0}
$$

\subsubsection{Application-level Delay}

Application-level Delay [39] $E D_{A}$ is the duration between the time when broadcast packets are generated at application layer of transmitting vehicle and the time at which the first successful packet is received by the application layer of receiving vehicle. Suppose the distance between the transmitting vehicle and the receiving vehicle is $r$, the average delay is given by

$E D_{A}(r)=\sum_{k=1}^{\infty}[E[D]+(i-1) t] P_{s}(r)\left(1-P_{s}(r)\right)^{i-1}=E[D]+t\left(\frac{1}{P_{s}(r)}-1\right)$

where $E[D]$ is the mean transmission delay of a packet, which is given in Eq. (20).

\section{Model Validation and Numerical Results}

In this section, we apply the proposed model to a typical broadcast network environment: DSRC (Dedicated short range communications) vehicular ad hoc communication system in a general 2-D area such as battlefield where soldiers are periodically informed about their updated situations or status [2-3]. Each node is equipped with IEEE 802.11 based wireless network capability with communication parameters as listed in Table I. Communication range (transmission/carrier sensing) is $R=500 \mathrm{~m}$. Each node generates broadcast messages with rate $\lambda$ and the average message length $E[P A]=200-400$ bytes. Two dimensional numerical integration in MATLAB is used to compute overlapping areas and impact of Nakagami fading needed for derivation of PDPs and PRRs. In order to cross-validate the proposed analytic model, we extend 1-D event-driven simulation program developed for research in [13] to 2-D simulation with Nakagami fading and path loss using both NS2 
Table 1: Parameters for Communication System

\begin{tabular}{|c|c|}
\hline Parameter & Value \\
\hline Modulation & BPSK, QPSK, 16-QAM, 64-QAM \\
\hline Coding Rates & $1 / 2,{ }^{2} / 3{ }^{3} / 4$ \\
\hline OFDM Symbol Duration & $8 \mu \mathrm{s}$ \\
\hline Signal Bandwidth & $10 \mathrm{MHz}$ \\
\hline Channel Data rate & $6,9,12,24,54 \mathrm{Mbit} / \mathrm{s}$ \\
\hline DIFS for 802.11a & $64 \mu \mathrm{s}$ \\
\hline Slot time $\sigma$ & $16 \mu \mathrm{s}$ \\
\hline SIFS for 802.11 & $32 \mu \mathrm{s}$ \\
\hline Propagation delay $\delta$ & $1 \mu \mathrm{s}$ \\
\hline Preamble Length & $40 \mu \mathrm{s}$ \\
\hline PLCP Header Length & $8 \mu \mathrm{s}$ \\
\hline CWMin & $15-1024$ \\
\hline
\end{tabular}

(version 2.35) and MATLAB. The communication nodes are Poisson distributed with density $\beta$ (nodes $/ \mathrm{m}^{2}$ ) on a 2-D area with radius of $3000 \mathrm{~m}$. Each node moves at speed of 10 meters per second with a random direction. In the NS2 program, the communication nodes are connected through calling IEEE 802.11 module Phy/WirelessPhyExt with transmission power 0.28183815 watts and receiving threshold 2.39455e-11 ((corresponding to 500 meters), and carrier sensing threshold up to 5.8e-12 (corresponding to 1000 meters), etc. The free space propagation model is assumed so that communication range is a random variable in the simulation. RF model in the simulation is set through module Antenna/OmniAntenna with gain of transmitters $G_{t}=1.0$ and with gain of receivers $G_{r}=1.0$. Nakagami channel with distance dependent path loss $(\gamma=2$, fading parameter $m$ is 3 for $d<50 m$, 1.5 for $50 m \leq d<150 m$, and 1 for $d>150 m$ ) is considered in the analysis and called in the simulation. The time resolution of the simulation program is exactly the minimum time unit $(1 \mu \mathrm{s})$ specified in IEEE 802.11 standard. This simulation procedure is performed multiple times based on independence replications, from which $95 \%$ confidence intervals (CIs) of the output measures can be computed.

Fig. 4 and Fig. 5 depict the packet reception ratios (PRRs) and packet transmission delay, respectively, over the average number of nodes in the 


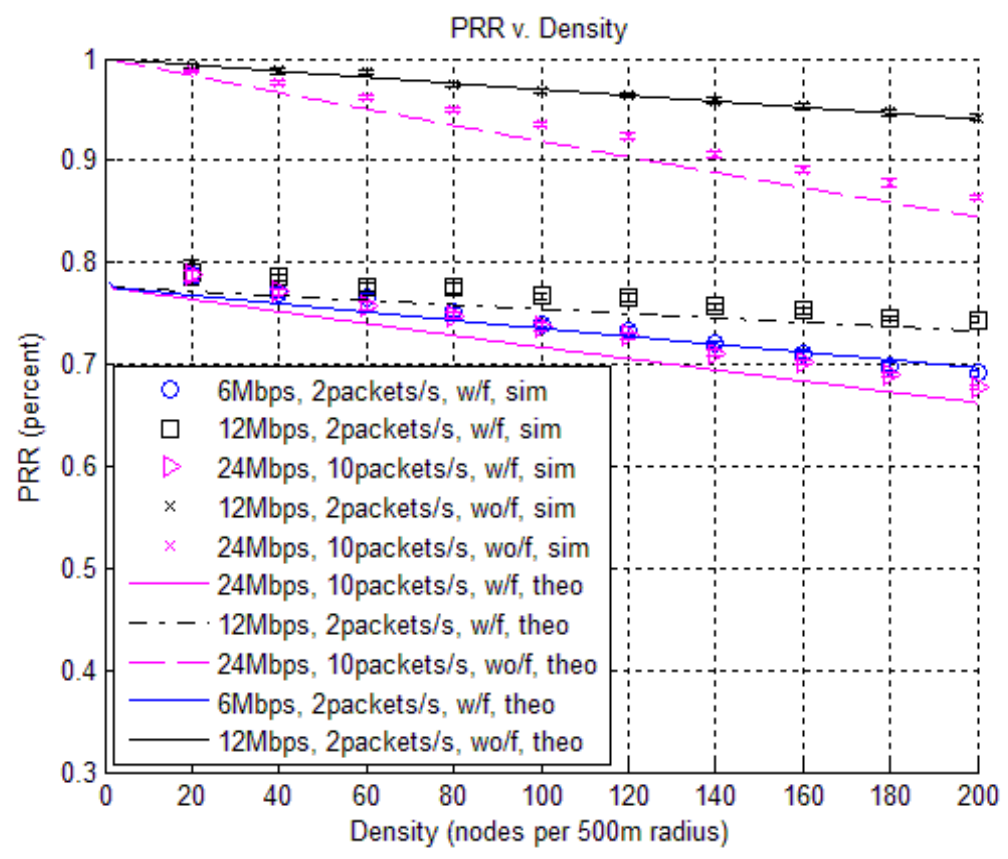

Figure 4: Packet reception ratios of vehicular broadcast, $W_{0}=15$

transmission range of a transmitting (or tagged) node. As shown in Fig. 4 and Fig. 5, analytical results (lines) practically coincide with the simulation results (symbols) with $95 \%$ CIs. The relative errors between the analytical results and the simulation results are $3.4 \%$ for $P R R s$, and $4.74 \%$ for $E[D] \mathrm{s}$. We also observe that PRRs get lower with the density of nodes in the network because more nodes introduce more data traffic on the ad hoc broadcast network. From Fig. 4, we can see that big difference between PRRs with Nakagami fading $(m=3)$ and PRRs without any fading, which indicates that, in addition to hidden terminal problem, fading is another major factor for degradation of reliability. We observe that channel fading does not affect the packet transmission delay. Also, it is observed that increasing transmission data rate (from $6 \mathrm{Mbps}$ to $12 \mathrm{Mbps}$ ) under inter-arrival packet generation rate 2 packets/second helps reduce average transmission delay.

Fig. 6 shows how Nakagami fading with different $m$ values affect PRRs of the ad hoc network broadcast communication. As stated earlier, fading degrades $P R R s$ significantly. PRRs with $m=3$ are bigger than PRRs with $m=1$ (Rayleigh distribution), which means bigger fading parameters bring 


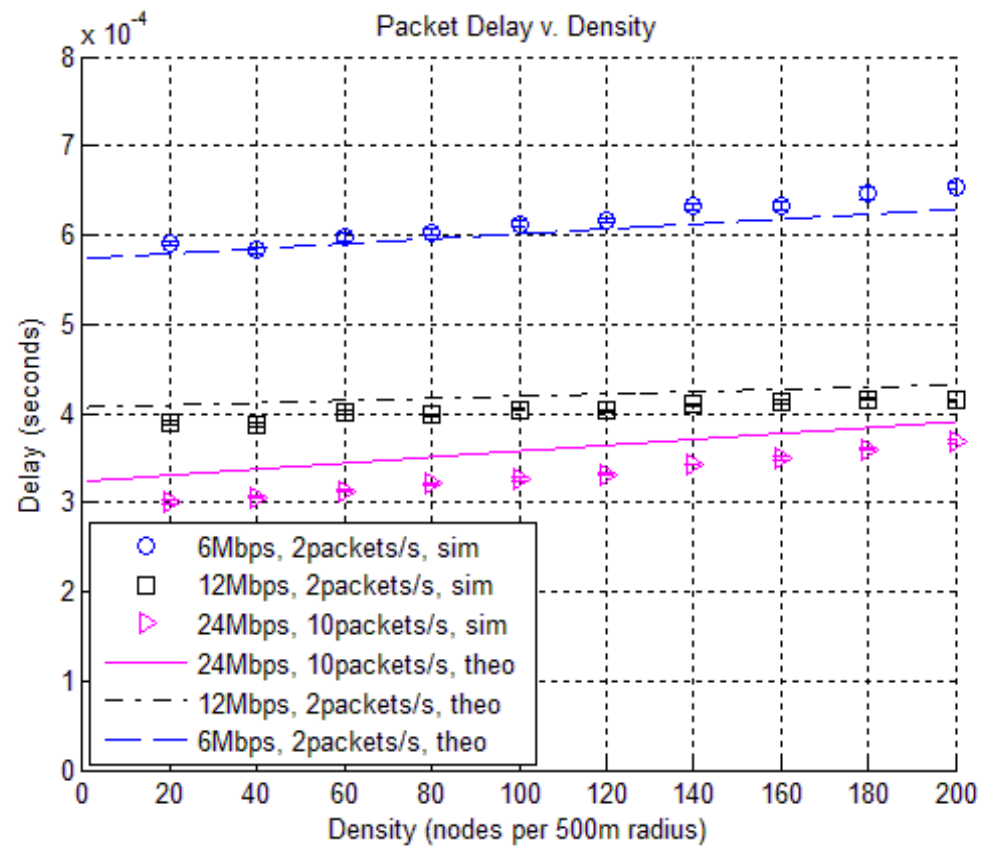

Figure 5: Packet transmission delay of vehicular broadcast, $W_{0}=15$

smaller fading to the communications.

Fig. 7 demonstrates how packet reception ratios (PRRs) and packet delivery probability $(P D P s)$ of a node change with the receivers' distances to the sender. Both PRRs and PDPs are reduced with the receiver 's distance to the sender, and are degraded with much higher rate as the receiver's distance to the sender is longer than $300 \mathrm{~m}$. The observation is due to the fact that the longer the receiver's distance to the sender, the more likely the receiver is affected by hidden terminal problem and channel fading. This observation alleviates the difficulty of selecting network parameters to meet the strict reliability requirements in some applications such as VANET safety message broadcast where high $P R R$ is required for the receiving nodes that are closer to the transmitting node. It is noticed that $P R R$ values and $P D P$ values are different because $P R R(d)$ is an average of $P D P$ values over area within radius $d$.

Fig. 8 shows how PDPs are affected by respective factors listed as hidden terminal problem, concurrent collisions, and channel fading. Given the same network parameters, separate PDP curves ignoring individual factors 


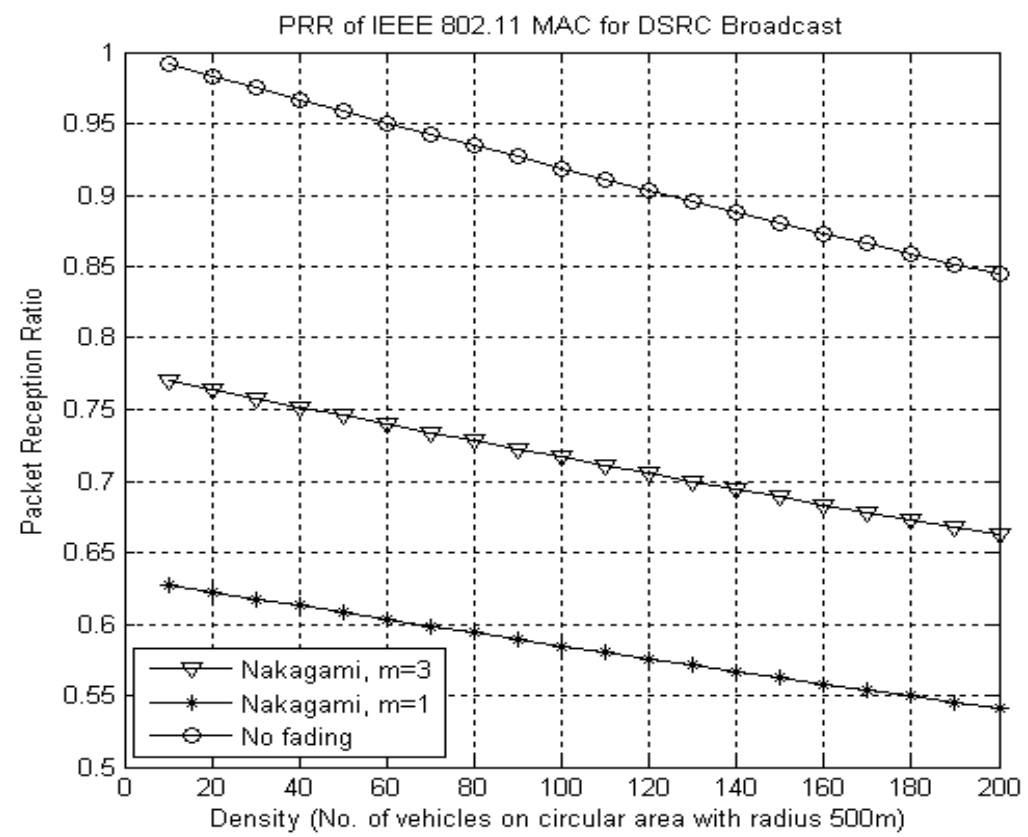

Figure 6: Impact of Nakagami fading on Packet reception rates of DSRC broadcast with network parameters $W_{0}=15, \lambda=10 \mathrm{pckts} / \mathrm{s}, R_{d}=24 \mathrm{Mbps}$

are compared with $P D P s$ taking all factors into account. It is observed that the curve without accounting for Nakagami fading has the biggest difference from the curve taking all factors into account among the other two curves, and the curve without accounting for concurrent collisions has the smallest difference from the curve taking all factors into account among the other two curves. This observation reveals that fading channel and hidden terminals problem are major factors to degrade the broadcast reliability, while concurrent collisions affect the reliability slightly.

Fig. 9 depicts the impact of communication ranges on the reliability with certain network setting. When carrier sensing range increases from $500 \mathrm{~m}$ to $1000 \mathrm{~m}$ (means w/o hidden terminals), PRRs increase accordingly because the bigger the carrier sensing range, the less the number of hidden terminals. It is interesting to see that the impact of fading is more significant than the impact of hidden terminals. If the interference range increases (from $500 \mathrm{~m}$ to $600 \mathrm{~m}), P R R s$ are getting worse even though carrier sensing range is extended (from $500 \mathrm{~m}$ to $800 \mathrm{~m}$ ). 


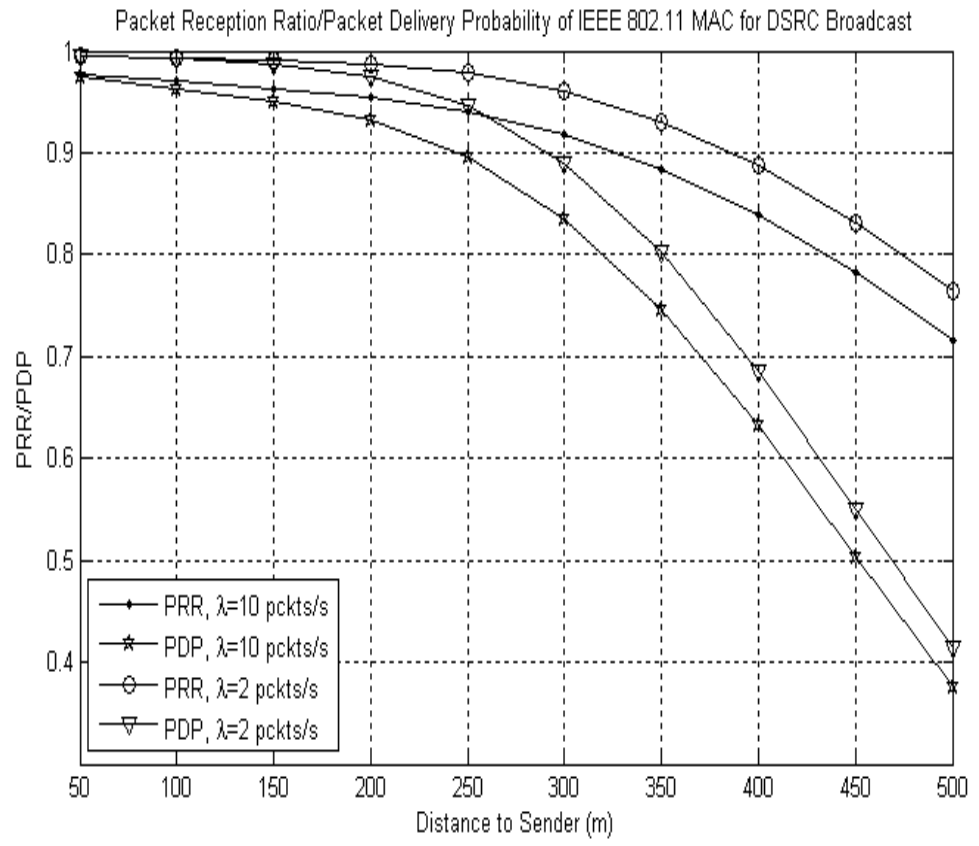

Figure 7: Packet reception ratios/packet delivery probabilities of DSRC broad-cast with network parameters $W_{0}=15, R_{d}=24 \mathrm{Mbps}, \beta=100 /\left(\pi R^{2}\right)$

Fig. 10 and Fig. 11 depicts analytic results for both MAC-level and Application-level reliability and packet transmission delay metrics under a given network parameter setting: Transmission range $R=500 \mathrm{~m}$, Time window $T_{w}=1 \mathrm{~s}$, Data rate $R_{d}=12 \mathrm{Mbps}$, Packet length $P L=200$ bytes, message generation interval $t=1 / \lambda=0.1 \mathrm{~s}$, node density $\beta=100 /\left(R^{2}\right)$ nodes $/ \mathrm{m} 2$. From Fig. 10, we can observe that all packet reception reliability metrics decrease with the distance from the sender. During the time window $T_{w}=1 \mathrm{~s}$, there are 10 packets sent out from the sender since the message interval is 0.1s. It is inspired to observe that although MAC-level broadcast reliability (PDP and PRR) is poor as the communication distance gets bigger, the application-level reliability can still be high enough. From Fig. 11, we can see that the application-level transmission delay is much higher than the MAC-level transmission delay. Fortunately, all the delays are less than $350 \mathrm{~ms}$, which meets the delay requirements of almost all applications. 


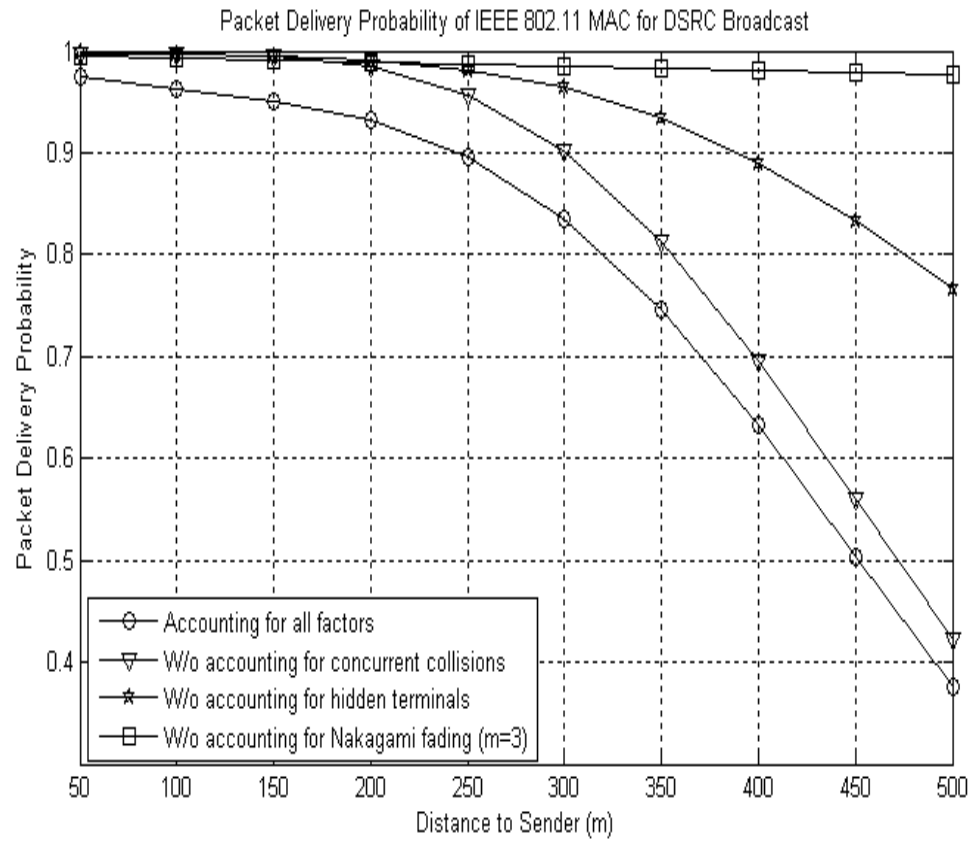

Figure 8: Packet delivery probabilities of DSRC broadcast with network parameters $\lambda=10$ pckts $/ \mathrm{s}, W_{0}=15, R_{d}=24 \mathrm{Mbps}, \beta=100 /\left(\pi R^{2}\right)$

\section{Conclusions}

In this paper, we propose an analytical model to evaluate the performance of IEEE 802.11 based broadcast two-dimensional wireless networks. A semi-Markov process model interacts with $\mathrm{M} / \mathrm{G} / 1 / \mathrm{K}$ queue model to characterize the behavior of communicating nodes under IEEE 802.11 broadcast. The derived performance and reliability metrics expressions from perspective of both MAC-level and Application-level take into account the impact of Nakagami fading channel with path loss, hidden terminals problem, concurrent transmissions, unsaturated message arrivals and varied communication ranges of IEEE 802.11 MAC, and DCF backoff process. As an example, the analytical model is applied to a vehicular ad hoc system for the broadcast of safety messages. PDP derivation using point-to-point integration facilitates the accurate impact analysis of fading channel. Numerical results prove the effectiveness and correctness of the proposed model, and reveal the characteristics of 2-D broadcast ad hoc networks, which can be beneficial to the design of such systems. From the numerical results and discussions, some 


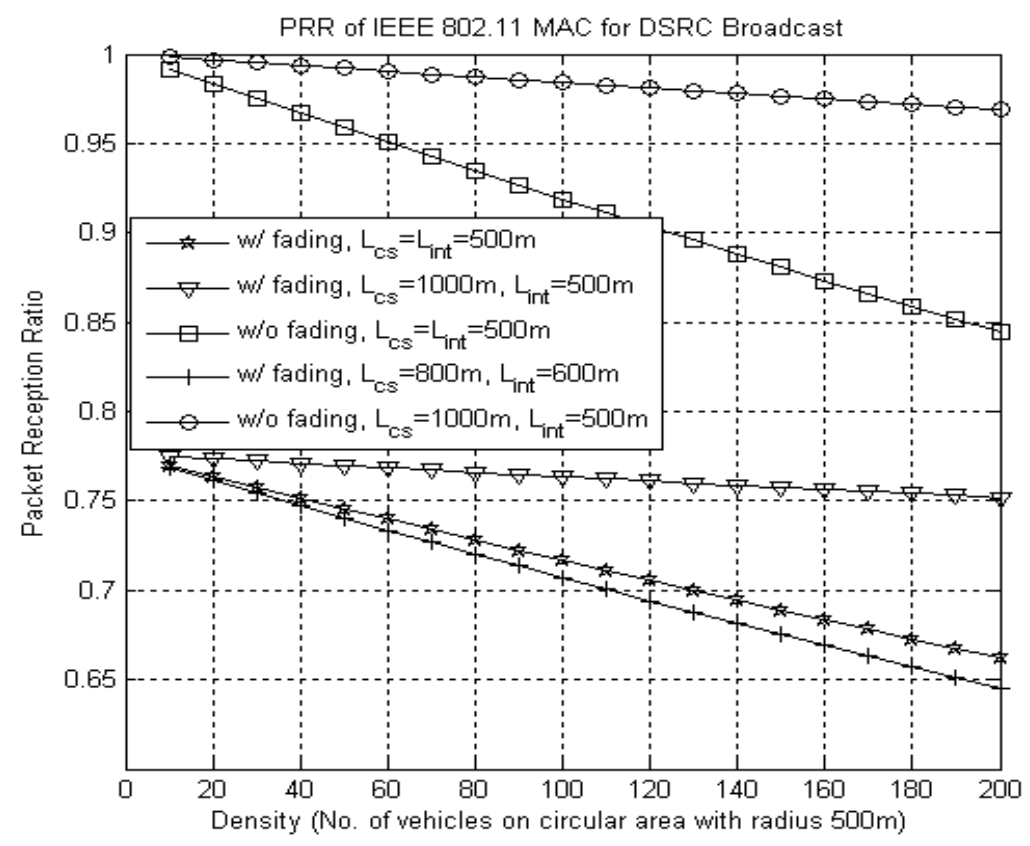

Figure 9: Impact of Communication range on Packet reception rates of DSRC broadcast with network parameters $W_{0}=15, \lambda=10$ pckts $/ \mathrm{s}, R_{d}=24 \mathrm{Mbps}$

very important observations of such networks are described.

Our future research will focus on building more specific models to fit various road conditions such as intersections, streets of cities, etc., and extend the proposed 2-D model to the models with more general node distributions and more general packet arrivals. It is noticed that the proposed model in this paper can be extended to address multiple services in multiple channels in a similar way described in [30] and [31].

\section{Appendix A. Derivation of $\left|S_{1}\left(r_{0}\right)\right|$ in Eq. (21)}

As shown in Fig. A.12, given distance $r_{0}\left(r_{0}<L_{c s}\right)$ between center $O$ for circle $D\left(O, L_{c s}\right)$ and center $Q$ for circle $D\left(Q, L_{i n t}\right)$, intersection points of two circles denoted as $I_{1}$ and $I_{2}$, and $X$ the range on $x$ axis projected by line $I_{1} O$, we have equation:

$$
\sqrt{L_{c s}^{2}-X^{2}}=\sqrt{L_{i n t}^{2}-\left(r_{0}-X\right)^{2}}
$$




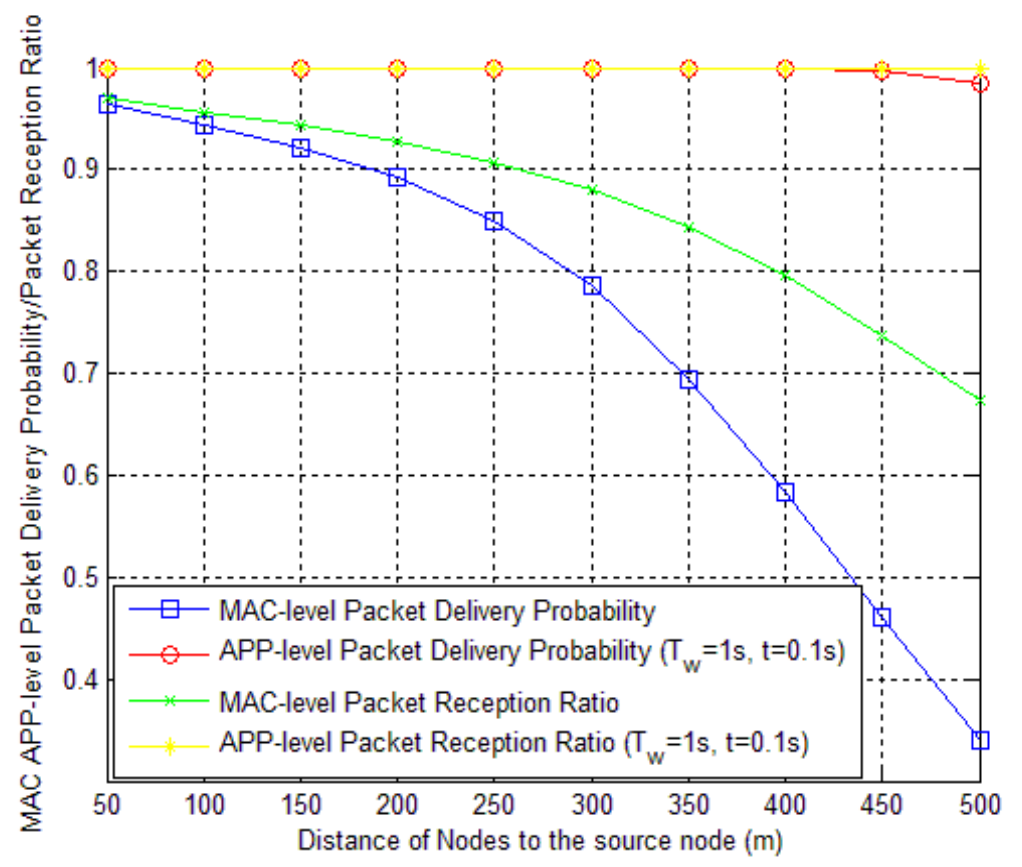

Figure 10: MAC (APP)-level PDP and PRR of DSRC broadcast with network parameters $W_{0}=15, \lambda=10 \mathrm{pckts} / \mathrm{s}, R_{d}=12 \mathrm{Mbps}, \beta=100 /\left(\pi R^{2}\right)$

Solving the above equation, we obtain

$$
X=\frac{L_{c s}{ }^{2}+r_{0}{ }^{2}-L_{i n t}{ }^{2}}{2 r_{0}}
$$

Let $\psi_{1}, \psi_{2}$ be radian of $I_{1} O$ and $I_{1} Q$, respectively. Then

$$
\begin{gathered}
\psi_{1}=\arccos \left(\frac{X}{L_{c s}}\right)=\arccos \left(\frac{\left.{L_{c s}^{2}+r_{0}^{2}-L_{i n t}^{2}}_{2 L_{i n t} r_{0}}\right)}{\psi_{2}=\arccos \left(\frac{r_{0}-X}{L_{i n t}}\right)=\arccos \left(\frac{L_{i n t}^{2}+r_{0}^{2}-L_{c s}^{2}}{2 L_{i n t} r_{0}}\right)}\right.
\end{gathered}
$$

Denote $\left|S_{1}^{\prime}\left(r_{0}\right)\right|$ as the overlapping area of $D\left(O, L_{c s}\right)$ and $D\left(Q, L_{i n t}\right)$, we have

$$
S_{1}^{\prime}\left(r_{0}\right)=L_{c s}^{2} \psi_{1}+L_{i n t}^{2} \psi_{2}-r_{0} \sqrt{L_{c s}^{2}-X^{2}}
$$

So,

$$
\left|S_{1}\left(r_{0}\right)\right|=\pi L_{i n t}^{2}-\left|S_{1}^{\prime}\left(r_{0}\right)\right|
$$




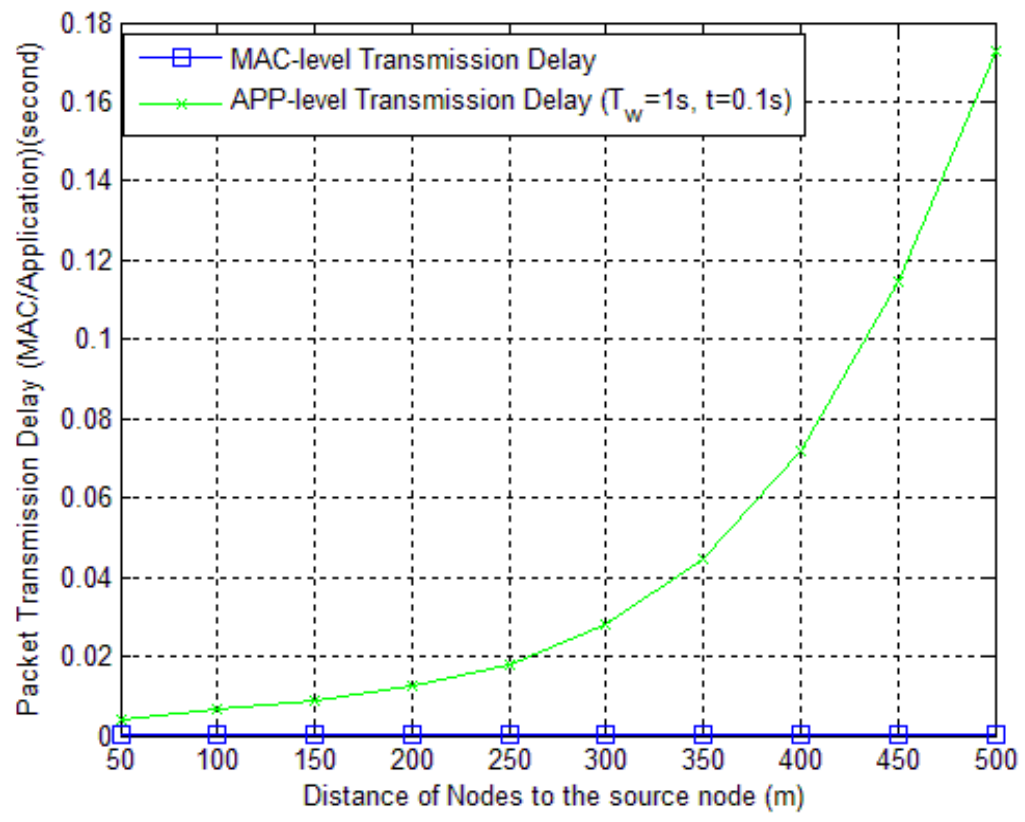

Figure 11: MAC (APP)-level Transmission delay of DSRC broadcast with network parameters $W_{0}=15, \lambda=10 \mathrm{pckts} / \mathrm{s}, R_{d}=12 \mathrm{Mbps}, \beta=100 /\left(\pi R^{2}\right)$

\section{Appendix B. Computation of $\left|S_{2}\left(\phi, r_{0}, r_{1}\right)\right|$ in Eq. (22)}

Given coordinates of nodes $P:(0,0), Q:\left(r_{0}, 0\right)$, and $V:\left(r_{1} \cos \phi, r_{1} \sin \phi\right)$, the contour functions of the transmission (or receiving) area of the nodes are: $D\left(O, L_{c s}\right): x^{2}+y^{2}=L_{c s}^{2}, D\left(Q, L_{i n t}\right):\left(x-r_{0}\right)^{2}+y^{2}=L_{i n t}{ }^{2}, D\left(V, L_{c s}\right):$ $\left(x-r_{1} \cos \phi\right)^{2}+\left(y-r_{1} \sin \phi\right)^{2}=L_{c s}{ }^{2}$.

Solving the above contour equations, we find the coordinates of all the intersection points $I_{P Q}{ }^{1}, I_{P Q}{ }^{2}, I_{P V}{ }^{1}, I_{P V}{ }^{2}, I_{Q V}{ }^{1}$, and $I_{Q V}{ }^{2}$. Only three out of six intersection points determine the shape of outmost contour. These intersection points can be selected through the following criterion: if the given intersection point $\left(x^{\prime}, y^{\prime}\right)$ is one of the outmost intersection points, the 


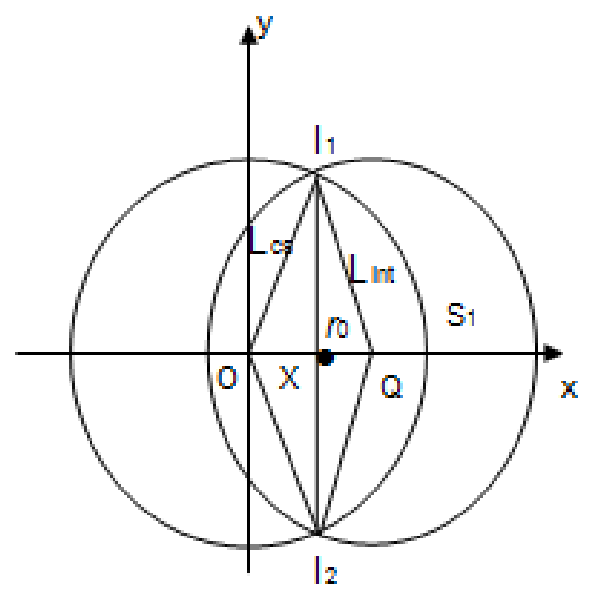

Figure A.12: Illustration of $S_{1}$ area calculation

following inequities must hold

$$
\begin{gathered}
x^{\prime 2}+y^{\prime 2} \geq L_{c s}{ }^{2} \\
\left(x^{\prime}-r_{0}\right)^{2}+y^{\prime 2} \geq L_{i n t}{ }^{2} \\
\left(x^{\prime}-r_{1} \cos \phi\right)^{2}+\left(y^{\prime}-r_{1} \sin \phi\right)^{2} \geq L_{c s}{ }^{2}
\end{gathered}
$$

It is easy to prove that the criterion is both sufficient and necessary.

By knowing the outmost intersection points and corresponding outmost curves, we are able to calculate the area surrounded by the outmost contour. Two neighboring outmost intersection points determine one outmost curve. Outmost curves all together form the outmost contour. In Fig. B.13, the outmost contour consists of the following curves:

Given the three outmost intersection points $I_{1}$ with coordinate $\left(x_{1}, y_{1}\right), I_{2}$ with coordinate $\left(x_{2}, y_{2}\right)$, and $I_{3}$ with coordinate $\left(x_{3}, y_{3}\right)$, whose radian coordinates are $\left(r_{i 1}, \theta_{1}\right),\left(r_{i 2}, \theta_{2}\right)$, and $\left(r_{i 3}, \theta_{3}\right)$, respectively, where $r_{i 1}=r_{i 3}=L_{c s}$; $r_{i 2}=\left(x_{i 2}^{2}+y_{i 2}^{2}\right)^{1 / 2} ; \theta_{1}=\arctan \left(\frac{y_{i 1}}{x_{i 1}}\right), \theta_{2}=\arctan \left(\frac{y_{i 2}}{x_{i 2}}\right), \theta_{3}=\arctan \left(\frac{y_{i 3}}{x_{i 3}}\right)$.

Outmost curves connected by $I_{1}, I_{2}$, and $I_{3}$ divide the total area into two sections: section $1\left(\widehat{I_{1} I_{2}}\right)$ center $V$ and section $2\left(\widehat{I_{2} I_{3}}\right)$ center $Q$, as shown in Fig. B.13. Areas of the two sections are denoted as $A_{1}$ and $A_{2}$, respectively. Given $O$ at the origin, radian of section 1 ranges from $\theta_{2}$ to $\theta_{1}$; radian of section 2 ranges from $\theta_{3}$ to $\theta_{2}$; and radian of $\widehat{V O}$ is $\phi$.

Given that $P$ is a point on the outmost curve of section 1 and the radian of $\widehat{P O}$ is $\theta$, the area of section 1 is 


$$
\begin{aligned}
A_{1} & =\frac{1}{2} \int_{\theta_{2}}^{\theta_{1}}|\widehat{P O}|^{2} d \theta \\
& =\frac{1}{2} \int_{\theta_{2}}^{\theta_{1}}\left(r_{1} \cos (\theta-\varphi)+\sqrt{L_{c s}^{2}-r_{1}^{2} \sin ^{2}(\theta-\varphi)}\right)^{2} d \theta
\end{aligned}
$$

So, $A_{1}$ can be expressed in Eq.(B.1).

$$
\begin{aligned}
A_{1}= & \frac{\left(L_{c s}^{2}-r_{1}^{2}\right)\left(\theta_{1}-\theta_{2}\right)}{2}+r_{1}^{2}\left[\frac{\theta-\phi}{2}+\frac{\sin 2(\theta-\phi)}{4}\right]- \\
& r_{1}{ }^{2} \frac{\sin (\theta-\phi) \sqrt{\left(\frac{L_{c s}}{r_{1}}\right)^{2}-\sin ^{2}(\theta-\phi)}}{2}-\left.\frac{L_{c s}^{2} \arcsin \left(\frac{r_{1} \sin (\theta-\phi)}{L_{c s}}\right)}{2}\right|_{\theta_{2}} ^{\theta_{1}}
\end{aligned}
$$

Given that $U$ is a point on the outmost curve of section 2 and the radian of $\widehat{U O}$ is $\theta$, the area of section 2 is

$$
\begin{aligned}
A_{2} & =\frac{1}{2} \int_{\theta_{3}}^{\theta_{2}}|\widehat{U O}|^{2} d \theta \\
& =\frac{1}{2} \int_{\theta_{3}}^{\theta_{2}}\left(r_{0} \cos \theta+\sqrt{L_{\text {int }}{ }^{2}-r_{0}^{2} \sin ^{2} \theta}\right)^{2} d \theta
\end{aligned}
$$

So, $A_{2}$ can be expressed in Eq.(B.2).

$$
\begin{aligned}
A_{2}=\frac{\left(L_{i n t}^{2}-r_{0}^{2}\right)\left(\theta_{2}-\theta_{3}\right)}{2}+r_{0}^{2}\left[\frac{\theta}{2}+\frac{\sin 2(\theta)}{4}\right]- \\
r_{0}{ }^{2} \frac{\sin (\theta) \sqrt{\left(\frac{L_{i n t}}{r_{0}}\right)^{2}-\sin ^{2}(\theta)}}{2}-\left.\frac{L_{i n t}{ }^{2} \arcsin \left(\frac{r_{0} \sin (\theta)}{L_{\text {int }}}\right)}{2}\right|_{\theta_{3}} ^{\theta_{3}}
\end{aligned}
$$

Then, the extended coverage area $S_{2}\left(\phi, r_{1}, r_{0}\right)$, as shown in Fig. B.13, can be calculated as the sum of $A_{1}$ and $A_{2}$ excluding the area of $D(O, R)$ and node $O$ s hidden terminal area in $A_{1}$ and $A_{2}$, which is expressed as

$$
S_{2}\left(\phi, r_{1}, r_{0}\right)=A_{1}+A_{2}-\frac{1}{2}\left(\theta_{1}-\theta_{3}\right) L_{c s}^{2}-\left|S_{1}\left(r_{0}\right)\right|
$$




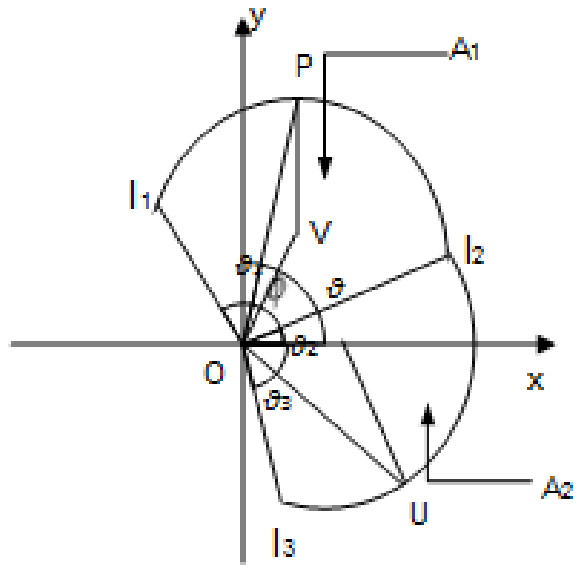

Figure B.13: Illustration of $S_{2}$ area calculation

\section{Appendix C. Solution to the embedded Markov chain}

To solve the following embedded Markov chain

$$
\left\{\begin{array}{c}
\nu P=\nu \\
\sum_{k=0}^{K-1} \nu_{k}=1
\end{array}\right.
$$

An efficient algorithm for computing can be given in terms of [25]

$$
\nu_{k}{ }^{d}=\frac{\nu_{k}}{\nu_{0}} \quad 0 \leq k \leq K-1
$$

Then $\nu^{d}$ can be recursively calculated as follows [25]:

$$
\begin{aligned}
\nu_{0}^{d} & =1 \\
\nu_{k+1}{ }^{d} & =\frac{1}{\alpha(0)}\left(\nu_{k}{ }^{d}-\sum_{j=1}^{k} \nu_{j}^{d} \alpha(k-j+1)-\alpha(k)\right) \quad 0 \leq k \leq K-2
\end{aligned}
$$

where $\alpha(k)$ is given by Eq. (15), and

$$
\nu_{0}=\frac{1}{\sum_{k=0}^{K-1} \nu_{k}^{d}} \quad \nu_{k}=\nu_{k}^{d} \nu_{0} \quad 0 \leq k \leq K-1
$$




\section{Acknowledgment}

The authors would like to thank NSF grants (CNS-1018605 and CNS1017722) to support this research. Also, the authors would like to thank Dr. Xianbo Chen, Matthew Wilson, and Gregory Butron for simulations \& 2-D coverage area computation.

\section{References}

[1] "Standard Specification for Telecommunications and Information Exchange Roadside and Vehicle Systems- 5GHz Band Dedicated Short Range Communications (DSRC) Medium Access Control (MAC) and Physical Layer (PHY) Specifications", April 2012.

[2] I.F. Akyildiz, W. Su, Y. Sankarasubramaniam, E. Cayirci, Wireless sensor networks: a survey, Computer Networks, Elsevier, 38 (2002) 393-422.

[3] C. Rajabhushanam and A. Kathirvel, Survey of wireless MANET application in battlefield operations, International Journal of Advanced Computer Science and Applications, 2(1), Jan. 2011.

[4] D. Huang, Measure for IEEE 802.11 based MANETs, IEEE Trans. on Wireless Communications, 7(3): 1025-1034, March, 2008.

[5] C. H. Foh, M. Zukerman, J. W. Tantra, A Markovian framework for performance evaluation of IEEE 802.11, IEEE Trans. on Wireless Communications, 6(4): 1276-1285, April, 2007.

[6] DARPA. Army collaborative future combat system FCS, http://www.darpa.mil/fcs

[7] B. M. Mughal, A. A. Wagan, H. Hasbullah, Measurement of single-hop periodic communication in vehicular environment, International Journal on New Computer Architectures and Their Applications, 1(3): 542-552, 2011 .

[8] X. Ma, Xianbo Chen, and Hazem Refai, On the broadcast packet reception rates in one-dimensional MANETs, IEEE GLOBECOM, Nov. 30-Dec. 4, New Orleans, 2008. 
[9] X. Li, Thu D. Nguyen, and R. P. Martin, An analytic model predicting the optimal range for maximizing 1-hop broadcast coverage in dense wireless networks, Proceedings of the 3rd International Conferences on Ad-Hoc Networks \& Wireless, pp. 172-182, Vancouver, Canada, July 2004.

[10] J. Choi, J. So, and Y. Ko, Numerical analysis of IEEE 802.11 broadcast scheme in multihop wireless ad hoc networks, The 19th International Conference on Information Networking, pp. 1-10, 2005.

[11] F. Ye, et al., Efficiency and Reliability of One-Hop Broadcasting in Vehicular Ad Hoc Networks, IEEE J. Selected Areas in Communications, 29(1), Jan. 2011.

[12] X. Ma and X. Chen, Delay and Broadcast Reception Rates of Highway Safety Applications in Vehicular Ad Hoc Networks, IEEE INFOCOM2007 Workshop on Mobile Networks for Vehicular Environments, Anchorage, Alaska, May 6 12, 2007.

[13] X. Ma, J. Zhang, and T. Wu, Reconsider Broadcast Packet Reception Rates in One-Dimensional MANETs, IEEE GLOBECOM 10, Dec. 6-10, 2010, Miami.

[14] X. Ma, H. H. Refai, On the broadcast packet reception rates in twodimensional MANETs, IEEE ICC11, June 2011.

[15] X. Ma, On the Reliability and Performance of Real-Time One-Hop Broadcast MANETs, ACM/Springer Wireless Networks, 17(5): 13231337, May 2011.

[16] K. Tang, M. Gerla, MAC layer broadcast support in 802.11 wireless networks, Proc. of Military Communication Conference (MILCOM 2000), Los Angeles, 2000.

[17] J. G. Andrews, R. K. Ganti, M. Haenggi, N. Jindal, and S. Weber, A Primer on Spatial Modeling and Analysis in Wireless Networks, IEEE Communications Magazine, Nov. 2010. 48(11): 156-163, Nov. 2010.

[18] K. S. Trivedi, Probability and Statistics with Reliability, Queuing and Computer Science Applications, Second Edition, John Wiley \& Sons, INC., 2002. 
[19] M. Killat and H. Hartenstein, An empirical model for probability of packet reception in vehicular ad hoc networks, EURASIP Journal on Wireless Communications and Networking, Volume 2009.

[20] X. Yin, X. Ma and K. S. Trivedi, Performance Evaluation for DSRC Vehicular Safety Communication: A Semi-Markov Process Approach, International Conference on Communication Theory, Reliability, and Quality of Service, 2011.

[21] P. K. Singh and K. Lego, Comparative study of radio propagation and mobility models in vehicular adhoc network, International Journal of Computer Applications, 16(8), Feb. 2011.

[22] A. Jayasuriya, S. Perreau, A. Dadej, and S. Gordon, Hidden vs. Exposed Terminal Problem in Ad Hoc Networks, Proceedings of the Australian Telecommunication Networks and Application Conference, Sydney, Australia, 8-10, Dec. 2009.

[23] D. Hoang, and R. A. Iltis, Performance Evaluation of Multi-hop CSMA/CA Networks in Fading Environments, IEEE Trans. on Communications, 56(1): 112-125, Jan. 2008.

[24] T. S. Rappaport, Wireless Communications, Principles 83 Practice,, Prentice Hall, 1996.

[25] M. Ozdemir, and A. B. Mcdonald, An M/MMGI/1/K queuing model for IEEE 802.11 ad hoc networks, 1st ACM international workshop on Performance Evaluation of Wireless Ad Hoc, Sensor, and Ubiquitous Networks, pp.107-111, 2004.

[26] Y. Zheng, K. Lu, D. Wu, and Y. Fang, Performance analysis of IEEE 802.11 DCF in binary symmetric channels, IEEE GLOBECOM, 2005.

[27] X. Ma, Packet Reception Ratios in Two-Dimensional Broadcast Ad Hoc Networks, IEEE International Conference on Computing, Networking and Communications (ICNC 2012), Communication QoS and System Modeling Symposium, Maui, Hawaii, Jan. 30-Feb. 2, 2012.

[28] C. Guo, J. Zhou, P. Pawelczak, and R. Hekmat, Improving packet delivery ratio estimation for indoor ad hoc and wireless sensor networks, Proc. 
of the 6th IEEE Consumer Communications and Networking Conference (CCNC09), pp. 1-5, Jan. 2009.

[29] M. Abolhasan and T. Eyers, Sparse ad hoc networks for the desert, DKCRC Research Report, 2007.

[30] MG Rubinstein, IM Moraes, Campista, LHMK Costa, OCMB Duarte, A survey on wireless ad hoc networks, Springer Mobile and Wireless Communication Networks, 1-31, 2006.

[31] J. Zhao and R. Govindan, Understanding packet delivery performance in dense wireless sensor networks, Proc. of ACM SenSys, Los Angeles, CA, Nov. 5-7, 2003.

[32] X. Yin, X. Ma, and K. S. Trivedi, Performance and reliability evaluation of BSM broadcasting in DSRC with multi-channel schemes, IEEE Transactions on Computers, 63(12): 3101-3113, Dec. 2014.

[33] R. B. Cooper, Introduction to Queueing theory, 1st Ed., North-Holland Publishing Company, 1981.

[34] F. Akyildiz, D. Pompili, and T. Melodia, Underwater acoustic sensor networks: research challenges, Ad Hoc Networks, 3 (2005) 257279, Elsevier.

[35] G. Bianchi, Performance analysis of the IEEE 802.11 distributed coordination function, IEEE J. Sel. Areas Commun., vol. 18, no. 3, pp. 535-547, 2000 .

[36] M. Gribaudo, D. Manini, A. Nordio, C. F. Chiasserini, Transient Analysis of IEEE 802.15.4 Sensor Networks, IEEE Transactions on Wireless Communications 10(4): 1165-1175, 2011.

[37] C.F. Chiasserini, R. Gaeta, M. Garetto, M. Gribaudo, D. Manini, M. Sereno, Fluid models for large-scale wireless sensor networks, Performance Evaluation 64(7-8): 715-736 (2007)

[38] M. Haenggi, Stochastic Geometry for Wireless Networks, Cambridge University Press, 2012. 
[39] F. Bai, H. Krishnan, V. Sadekar, G. Holland, T. ElBatt, Towards characterizing and classifying communication-based automotive applications from a wireless networking perspective, Workshop on Autonet, 2006.

[40] P. Papadimitratos, A. La Fortelle, K. Evenssen, R. Brignolo, S. Cosenza, Vehicular communication systems: enabling technologies, applications, and future outlook on intelligent transportation, Communication Magazine, 47(11): 84-95, Nov. 2009. 